Note

\title{
Hormonal Regulation of Glycolytic Enzyme Gene and Pyruvate Dehydrogenase Kinase/Phosphatase Gene Transcription
}

\author{
Zhe WANG, YASUMASA IWASAKI, Li FENG ZHAO, MitsuRU NISHIYAMA, TAKAFUMI TAGUCHI, \\ MAKOTO TSUGITA, MACHIKO KAMBAYASHI, KOZO HASHIMOTO AND YOSHIO TERADA \\ Department of Endocrinology, Metabolism, and Nephrology, Kochi Medical School, Kochi University, Kohasu, Oko-cho, \\ Nankoku 783-8505, Japan
}

\begin{abstract}
Both glucocorticoid and insulin are known to have an anabolic effect on lipogenesis. The glycolytic pathway is a part of the lipogenic pathway in the liver, and glycolytic enzymes mediate the conversion from glucose to pyruvate, and pyruvate dehydrogenase complex (PDC) mediates the conversion from pyruvate to acetyl-CoA, the activity of which is regulated by pyruvate dehydrogenase kinases (PDKs) and phosphatases (PDPs). In this study, we surveyed the effects of glucocorticoid, insulin, and forskolin (used as a surrogate of glucagon) on the transcriptional activity of glucokinase (GK), phosphofructokinase-1 (PFK1), liver-type pyruvate kinase (LPK), and all the PDKs/PDPs isoform genes. We found that both glucocorticoid and insulin had positive effects on PFK1 and LPK, whereas on GK the two hormones showed the opposite effect. Regarding the PDKs/PDPs, glucocorticoid significantly stimulated the transcriptional activity of all PDKs, among which the effect on PDK4 was the most prominent. Insulin alone had minimal effects on PDKs, but dampened the positive effects of glucocorticoid. On PDPs, glucocorticoid and forskolin showed negative effects, whereas insulin had positive effects; insulin and glucocorticoid/forskolin antagonized each other. Altogether, our data suggest that both glucocorticoid and insulin have lipogenic effects through positive effects on PFK1 and LPK expression. However, glucocorticoid antagonizes the effect of insulin at the level of GK to maintain glucose homeostasis and that of PDKs/PDPs to facilitate gluconeogenesis. Glucagon may also enhance gluconeogenesis by inhibiting PDPs.
\end{abstract}

Key words: Glycolysis, Pyruvate dehydrognase kinase, Pyruvate dehydrogenase phosphatase, Glucocorticoid, Insulin

(Endocrine Journal 56: 1019-1030, 2009)

IN the liver, the glycolytic pathway is a part of the lipogenic/gluconeogenic pathways. Glucose is immediately phosphorylated by glucokinase (GK), a liver-specific isoform of hexokinases, and the product glucose-6-phosphate is supplied for glycogen synthesis, or metabolized into pyruvate via the glycolytic pathway including key enzymes such as phosphofructokinase-1 (PFK1) and liver-type pyruvate kinase (LPK). Pyruvate is then converted either to acetylCoA by pyruvate dehydrogenase complex (PDC) for fatty acid synthesis during a fed state, or to oxaloacetate by pyruvate carboxylase for gluconeogenesis dur-

Received Jun. 19, 2009; Accepted Jul. 27, 2009 as K09E-178 Released online in J-STAGE as advance publication Aug. 25, 2009 Correspondence to: Yasumasa IWASAKI, M.D., Ph.D., Department of Endocrinology, Metabolism, and Nephrology, Kochi Medical School, Kochi University, Kohasu, Oko-cho, Nankoku 783-8505, Japan. E-mail: iwasaki@kochi-u.ac.jp ing a fasting state. Thus, the fate of carbohydrate is regulated not only by glycolytic enzymes but also by PDC, the activity of which is under the control of a variety of glucoregulatory hormones and/or nutritional factors [1-3]. PDC is a large multi-subunit complex, which is known to be activated via dephosphorylation by pyruvate dehydrogenase phosphatases (PDPs) and inactivated via phosphorylation by pyruvate dehydrogenase kinases (PDKs) [2, 4, 5]. There are two PDP isoforms (PDP1, PDP2) and a regulatory subunit (PDPRs), and four PDK isoforms (PDK1-4) [6, 7].

The short-term regulation of glycolysis/lipogenesis/ gluconeogenesis is mediated by allosteric changes of the enzymes. However, recent studies suggest the importance of long-term regulation at the transcriptional level. Indeed, the amount of mRNA of the enzymes is known to change dynamically in response to both fasting and feeding, and insulin plays a pivotal role in 
this regulation after feeding. Glucocorticoid is also involved in glucose metabolism, especially during the fasting state [8]. Although the primary role of glucocorticoid in higher organisms is, as "gluco" literally means, the maintenance of blood glucose, glucocorticoid excess is known to cause abdominal obesity [9], and thus glucocorticoid is recognized as an anabolic hormone in terms of triglyceride metabolism. In addition, glucagon plays an important role in gluconeogenesis by increasing intracellular cAMP levels and suppressing the activities of glycolytic enzymes. Nevertheless, the roles of glucocorticoid and glucagon on the transcriptional regulation of glycolytic/lipogenic genes are much less characterized compared with that of insulin.

Based on this background, in this study, we comprehensively studied in vitro the transcriptional regulation of all the promoters of the key glycolytic enzymes (GK, PFK1, LPK) and all the PDK and PDP isoform genes involved in the regulation of PDC, to clarify the possible differential regulation of each gene by the glucoregulatory hormones such as insulin, glucocorticoid, and glucagon/cAMP.

\section{Materials and Methods}

\section{Reagents and plasmids}

Human insulin was provided by Eli Lilly (Indianapolis, IN, USA). Dexamethasone and forskolin were purchased from Sigma (St. Louis, MO, USA).

The 5'-promoter regions of the human GK (1.65 kb), PFK1 (0.8 kb), LPK (2 kb), PDK1 (3 kb), PDK2 (3 kb), PDK3 (3 kb), PDK4 (1 kb), PDP1 (1 kb), PDP2 (1.5 kb), and PDPRs (2 kb) were cloned by PCR and incorporated into the pA3Luc luciferase reporter plasmid using standard molecular biology techniques.

\section{Cell culture and experiments}

The human hepatocyte-derived HepG2 and HuH7 cells were maintained in high glucose DMEM (Sigma) supplemented with $10 \%$ fetal bovine serum (FBS) and penicillin/streptomycin under a $5 \% \mathrm{CO}_{2} / 95 \%$ air atmosphere at $37^{\circ} \mathrm{C}$. In each experiment, cells were plated and cultured in 24 -well plates to $50 \%$ confluency. On the next day, the cells were transfected simultaneously with each of the promoter-luciferase plasmids with both human insulin receptor (IR) and glucocorticoid receptor (GR) expression vectors [luciferase plasmid: GR: IR = 4:1:1 ( $\mu \mathrm{g})]$ using FuGene 6 transfection reagent (Roche, Indianapolis, IN, USA), and 24 h later the medium was changed to serum-free medium. In each experiment, test reagents, in 1000x concentration, were then added directly into the culture medium, and the cells were incubated for the defined time interval. At the end of incubation, the culture medium was removed, and the cells were harvested for the determination of luciferase activity. All the experiments were carried out using HepG2 cells, but HuH7 cells were also used in some experiments to confirm the consistency of the data.

\section{Luciferase assay}

Luciferase assay was performed as previously described [10], and light output was measured for $20 \mathrm{sec}$ at room temperature using a luminometer (Berthold Lumat LB9507, Bad Wildbad, Germany).

\section{RT-PCR}

Endogenous expression of GK, PFK1, and LPK mRNAs in HepG2 and HuH7 cells was examined by RT-PCR. The cells were plated in 3.5-cm-diameter dishes, and total RINA was extracted using RNeasy RNA extraction kit ((Qiagen, Hilden, Germany). One $\mu \mathrm{g}$ each was then applied for reverse transcription reaction using Superscript III reverse transcriptase (Invitrogen), followed by PCR reaction (25-30 cycles, $94^{\circ} \mathrm{C} 30 \mathrm{sec}$, $60^{\circ} \mathrm{C} 30 \mathrm{sec}, 72^{\circ} \mathrm{C} 2 \mathrm{~min}$ ) using Blend Taq DNA polymerase (Toyobo, Tokyo, Japan). The primer sets used were: GK (HepG2) sense 5'-CTTCGACTACATCTC TGAGTGC-3', antisense 5'-GTCATACTCCAG CAGGAACTC-3'; GK (HuH7) sense 5'-CAGA TCTACAACATCCTGAGC-3', antisense 5'-CA GAG TCTCTGTTCCTGCTC-3'; PFK1 sense 5'-GGAG AACTTCATGTGTGAGAG-3', antisense 5'-GAG AAGTTAGACTTCTCCTTGG-3'; LPK sense 5'-C TCGCAGTACCAGCATCATTG-3', antisense 5'-G AGGCAAAGACGATGTCCAC-3'; PDK1 sense 5'-G TCTCCTTCCAGATAATCT TCTC-3', antisense 5'-C ACCATGTGATAGAGATGGGATG-3'; PDK2 sense 5'CATCATGAAAGAGATCAACCTG-3', antisense 5'CATTCTTGAAGAGCTCAAAGAG-3'; PDK3 sense 5-GGACTTCGGGAGAGATAATGC-3', antisense 5'GCATCTTGGCTGTTTCATATGC-3'; PDK4 sense 5'GACAAGAATTGCCTGTGAGAC-3', antisense 5'- 

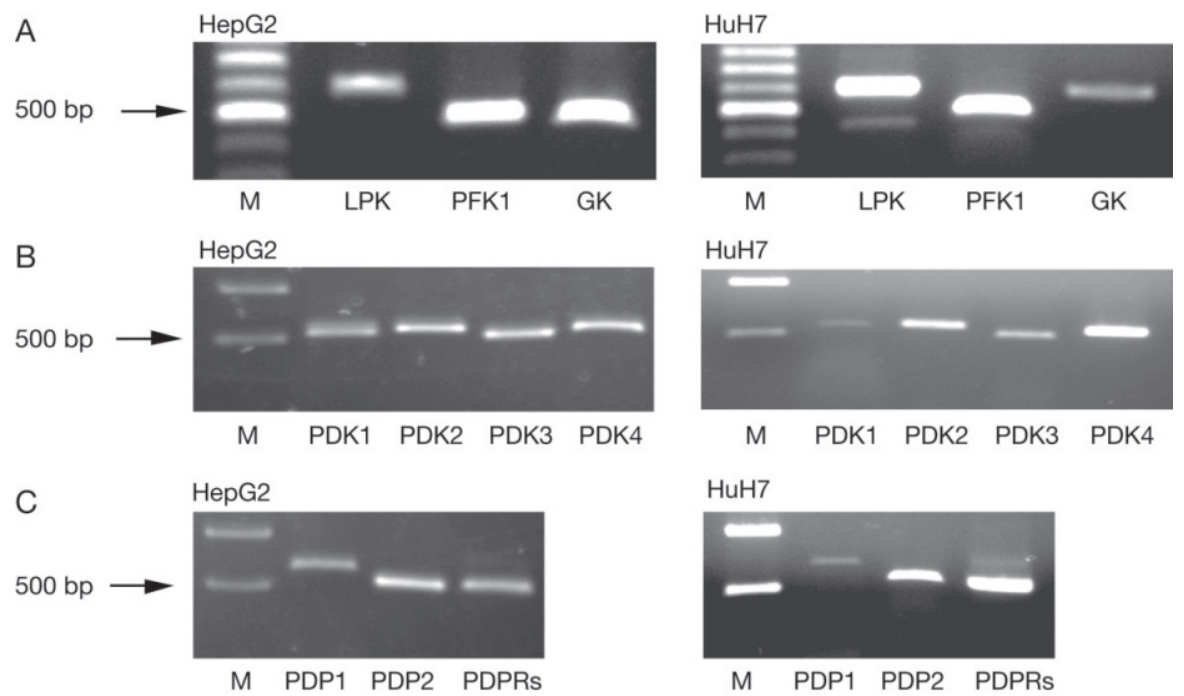

Fig. 1. RT-PCR analysis of the intrinsic expression of GK, PFK1, LPK, PDK1-4, PDP1, 2, and PDPRs mRNAs in HepG2 and HuH7 cells. The figure shows photographs of the ethidium bromide-stained products using agarose gel electrophoresis. cDNA produced from the RT reaction, using total RNA from the cells, was amplified by PCR with pairs of oligonucleotide primers specific for each mRNA. M: molecular size marker.

GAACATACACGATGTGAATTGG-3'; PDP1 sense 5'-CTAATGAATACAGTTTCAAAGTG-3', antisense 5'-GGTCATTAGACAGCGTGACTG-3'; PDP2 sense 5'-GTCACATCTGTGCATCTTGAC-3', antisense 5'GTGTGAACTGGTAAATGTTGAG-3'; and PDPRs sense 5'-GTCTGAAGTCAAGTGCTGTAAGG-3', antisense 5'-CTCATCACTTCATTGTATACATG-3'. The sequences for both sense and antisense primers in each mRNA were obtained from the different exon, to distinguish the mRNA-derived PCR product from that of the genomic DNA-derived one.

\section{Statistical Analysis}

Samples in each group of the experiments were in triplicate or quadruplicate. All data were expressed as a mean \pm SEM. When the statistical analyses were performed, data were compared by one-way analysis of variance with Fisher's PLSD test, and $p$ values below 0.05 were considered significant.

\section{Results}

Endogenous expression of glycolytic/lipogenic enzyme mRNAs in HepG2 and HuH7 cells

The intrinsic expression of mRNAs for GK, PFK1,
LPK, and each isoform of PDKs and PDPs was analyzed by RT-PCR. As shown in Fig. 1, mRNAs for all genes were amplified, indicating that both cell lines are appropriate for the transcriptional regulation of these genes in vitro.

Time-course effects of glucoregulatory hormones on the transcriptional activity of glycolytic/lipogenic enzyme genes

We first examined the effect of a synthetic glucocorticoid dexamethasone, insulin, and forskolin, used as a surrogate of glucagon, on the transcriptional activity of GK, PFK1, and LPK genes. As shown in Fig. 2, dexamethasone enhanced the 5'-promoter activity of PFK1 and LPK, and insulin enhanced that of all genes. In contrast, forskolin showed negative effects on the transcriptional activity of PFK1 and LPK genes.

Combined effects of insulin and dexamethasone on the expression of glycolytic/lipogenic enzyme genes

Since insulin and glucocorticoid have anabolic effects on lipogenesis, we then examined the combined effects of both hormones on the transcriptional activity of glycolytic/adipogenic enzyme genes. Dexamethasone alone, as observed in the above experiment, showed no effect on the transcriptional activity 
A

B

Promoter Activity

(RLU; \% control)

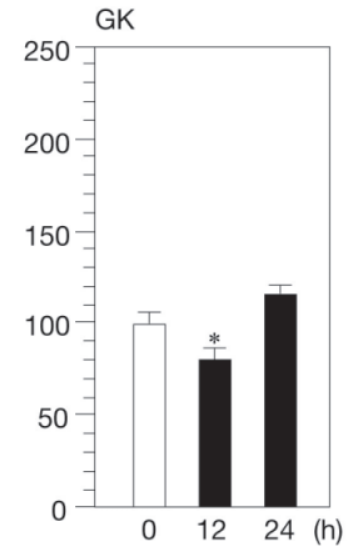

GK

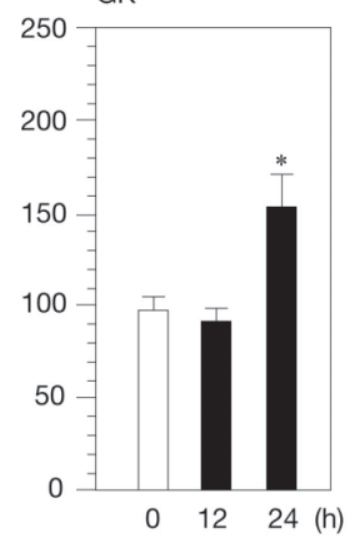

C

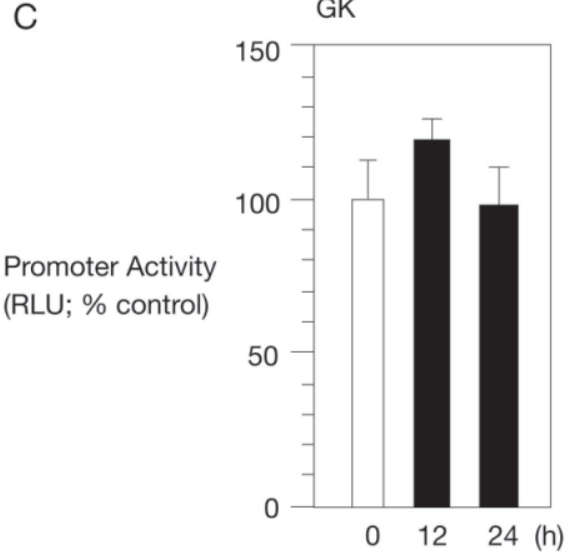

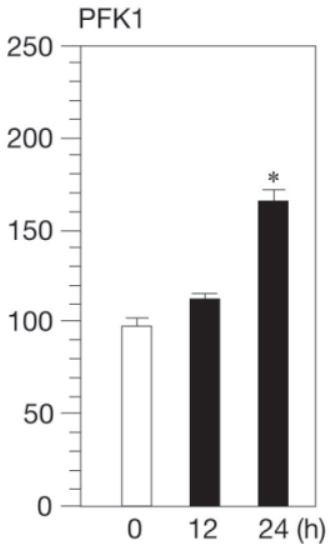
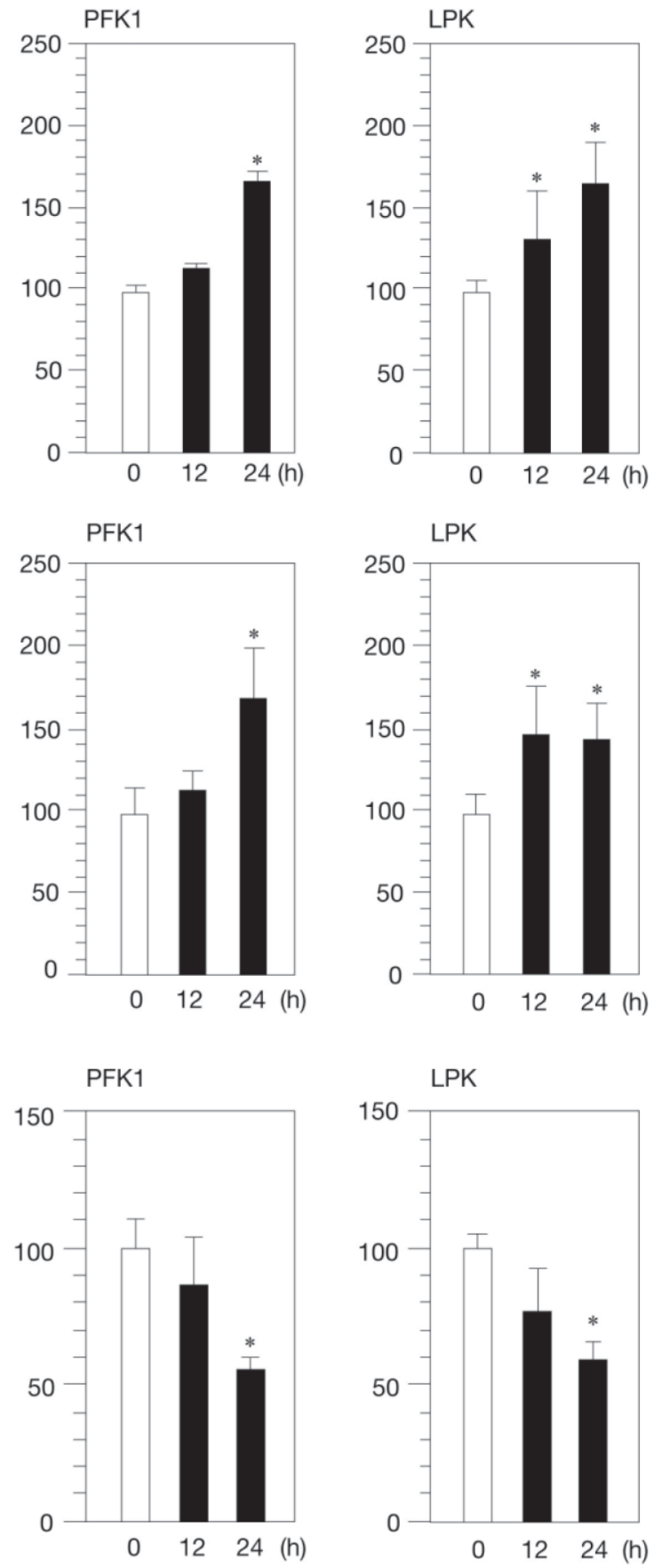

Fig. 2. Time-course effects of glucoregulatory hormones/factors on the transcriptional activity of GK, PFK1, and LPK. A-C. HepG2 cells were transfected transiently with each test plasmid and treated with dexamethasone (A) (100 nM), insulin (B) (10 nM), or forskolin (C) $(10 \mu \mathrm{M})$, for up to $24 \mathrm{~h}$. Each value is shown as a percentage of the value at time zero (0). RLU, Relative Light Unit. $* P<0.05$ vs. value at time zero.

of GK at 24 h. However, when used simultaneously with insulin, dexamethasone significantly suppressed the insulin-induced transcription, suggesting the presence of an antagonistic effect. In sharp contrast, both dexamethasone and insulin stimulated the LPK gene transcription in an additive fashion. Dexamethasone and insulin also increased PFK1 gene transcription, but no additional effect was observed. 

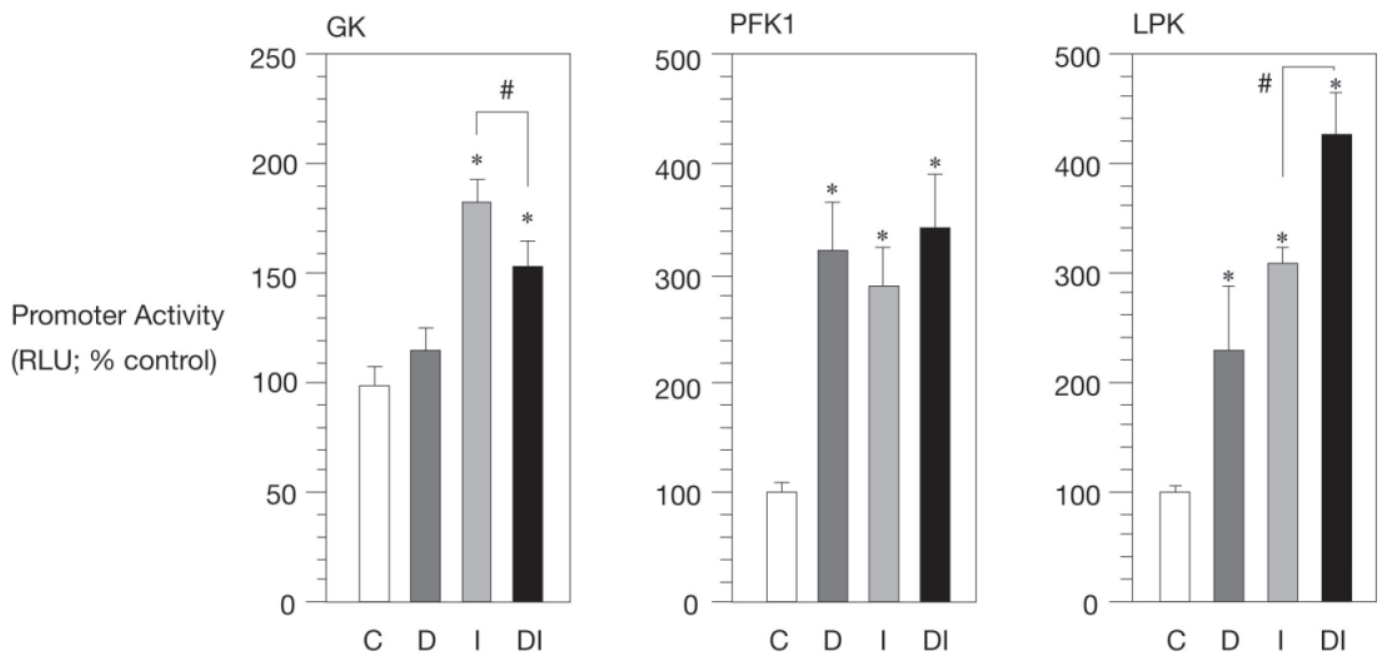

Fig. 3. Combined effects of dexamethasone and insulin on the transcriptional activity of GK, PFK1, and GK. HepG2 cells were transfected transiently with each test plasmid and treated with dexamethasone (D, $100 \mathrm{nM}$ ) and/or insulin (I, $10 \mathrm{nM}) \mathrm{for} 24 \mathrm{~h}$. Each value is shown as a percentage of the control value (C). RLU, Relative Light Unit. $* P<0.05$ vs. value at time zero. \# $P$ $<0.05$ vs. insulin alone.

Time-course and dose-response effects of glucoregulatory hormones on the transcriptional activity of each isoform of PDK genes

We next examined the time-course effects of hormones/factors on the transcriptional activity of PDK1-4 genes in HepG2 cells. Dexamethasone (100 $\mathrm{nM}$ ) showed significant stimulatory effects on the transcription of all the PDK isoform genes in a timedependent manner, among which the effect on PDK4 was most remarkable ( $\approx 800 \%$ increase) (Fig. 4A). Similar effects were observed in HuH7 cells. Doseresponse study also showed that a significant positive effect of dexamethasone (12 h) was observed in HepG2 cells at $10 \mathrm{nM}$ and was maximal at or above $100 \mathrm{nM}$ (Fig. 4B). On the other hand, insulin (10 nM) showed no or mild $(\approx 50 \%)$ stimulatory effect on the 5'-promoter activity of these genes in both HepG2 and HuH7 cells (Fig. 4C). Forskolin largely had no or minimal inhibitory effects on the 5'-promoter activity of PDK genes in both cell lines (data not shown).

Combined effects of the glucoregulatory hormones on the transcriptional activity of each isoform of PDK genes

We then examined the combined effects of glucocorticoid and other glucoregulatory hormones/factors on the transcriptional activity of each PDK isoenzyme genes in HepG2 cells. Dexamethasone (100 nM, 12 h) alone again showed robust and mild stimulatory effects on PDK4 and PDK1-3 gene transcription, respectively. However, when insulin was simultaneously used, the hormone significantly inhibited the dexamethasone-induced transcription (Fig. 5A). This antagonistic nature of insulin's effect on glucocorticoid was most remarkable in PDK4, but similar trends were also observed in other PDKs. Dose-response study showed that insulin exerted significant negative effects on dexamethasone-stimulated PDK4 gene transcription at or above $1 \mathrm{nM}$ (Fig. 5B). In contrast, forskolin did not influence the positive effects of glucocorticoid (Fig. 5C) or that of insulin (data not shown) on the gene transcription of all the PDK isoforms.

Time-course effects of glucoregulatory hormones on the transcriptional activity of each isoform of PDP genes

The effects of three glucoregulatory hormones/factors on the transcriptional activities of PDP1, 2, and PDPRs were also examined in HepG2 cells. In contrast to the positive effects on PDKs, dexamethasone significantly inhibited the basal transcriptional activities of PDP1 and 2 (Fig. 6A). Forskolin, which largely did not influence PDKs expression, also exerted potent inhibitory effects on the promoter activities of PDP1, 2 and PDPRs (Fig. 6C). In contrast, insulin showed positive effects on all the PDP isoforms (Fig. 6B). Similar effects were observed in HuH7 cells. 

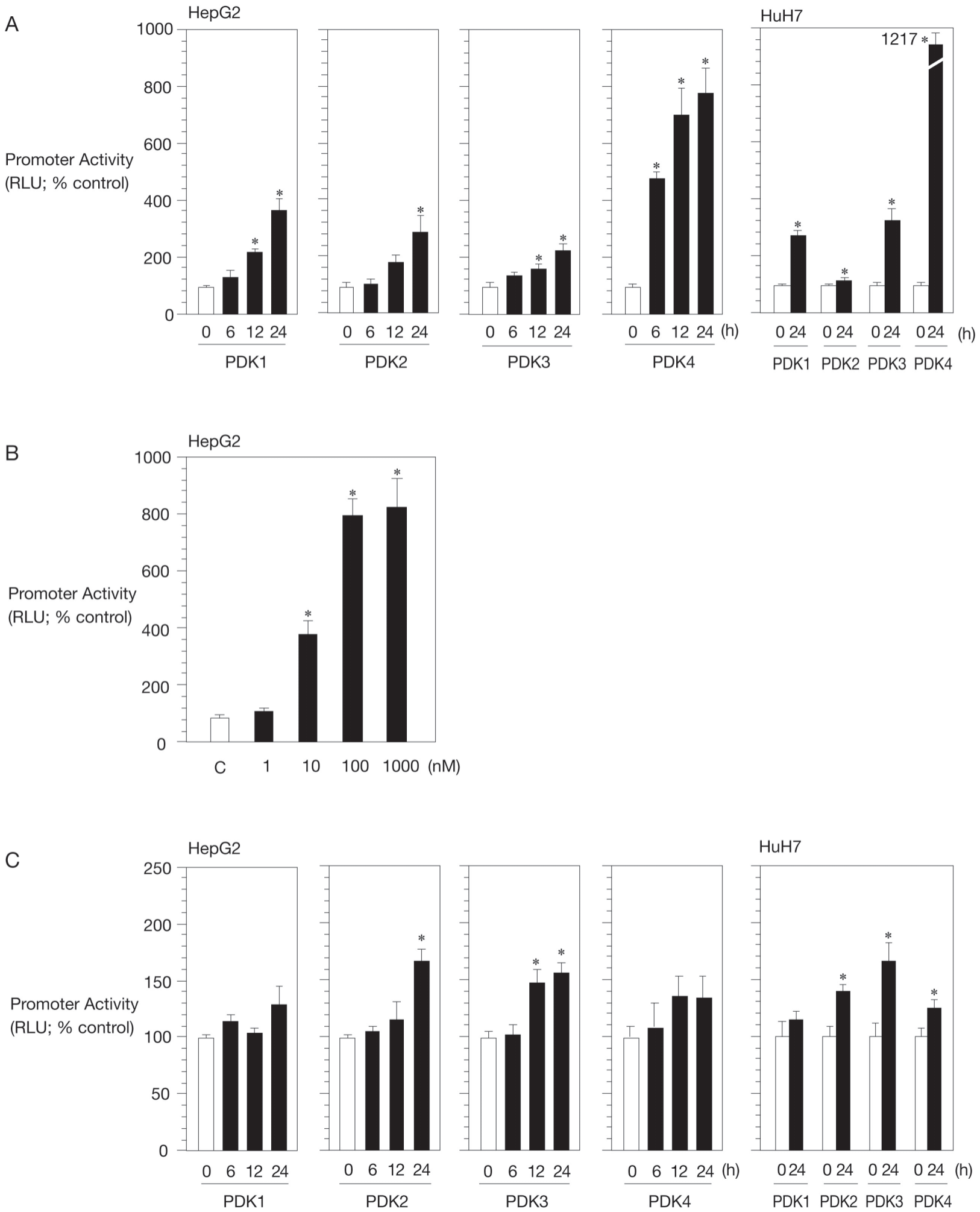

Fig. 4. Time-course and dose-response effects of glucocorticoid and insulin on the transcriptional activity of PDKs. A. HepG2 and HuH7 cells were transfected transiently with each test plasmid and treated with dexamethasone (100 nM) for 6-24 h (HepG2) or $24 \mathrm{~h}(\mathrm{HuH7})$. B. HepG2 cells were transfected as above and treated with various doses of dexamethasone (0, 1-1000 $\mathrm{nM})$ for $12 \mathrm{~h}$. C. Similar time-course experiments as A were carried out with insulin (10 nM). Each value is shown as a percentage of the value at time zero (0) or vehicle (C). RLU, Relative Light Unit. $* P<0.05$ vs. value at time zero. 
A

B

Promoter Activity

(RLU; \% control)

C

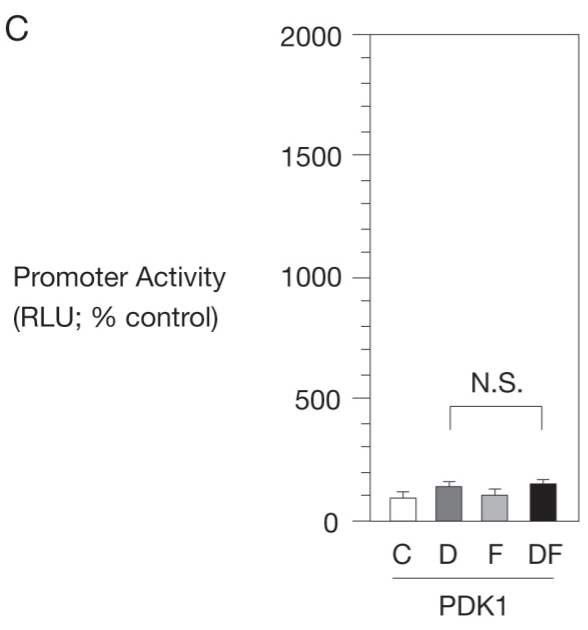

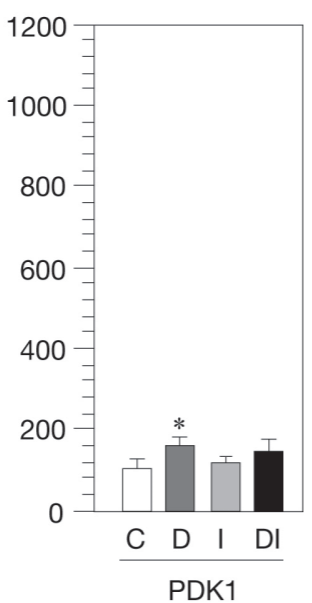
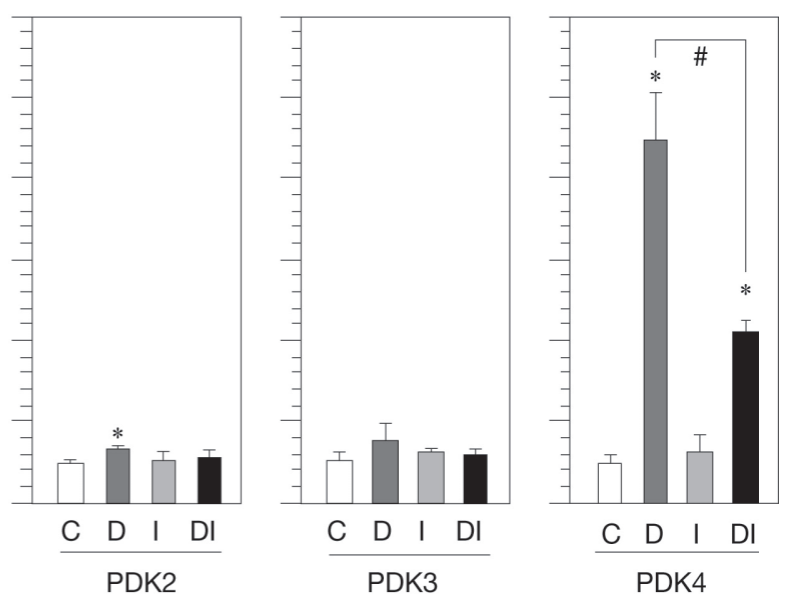

PDK4
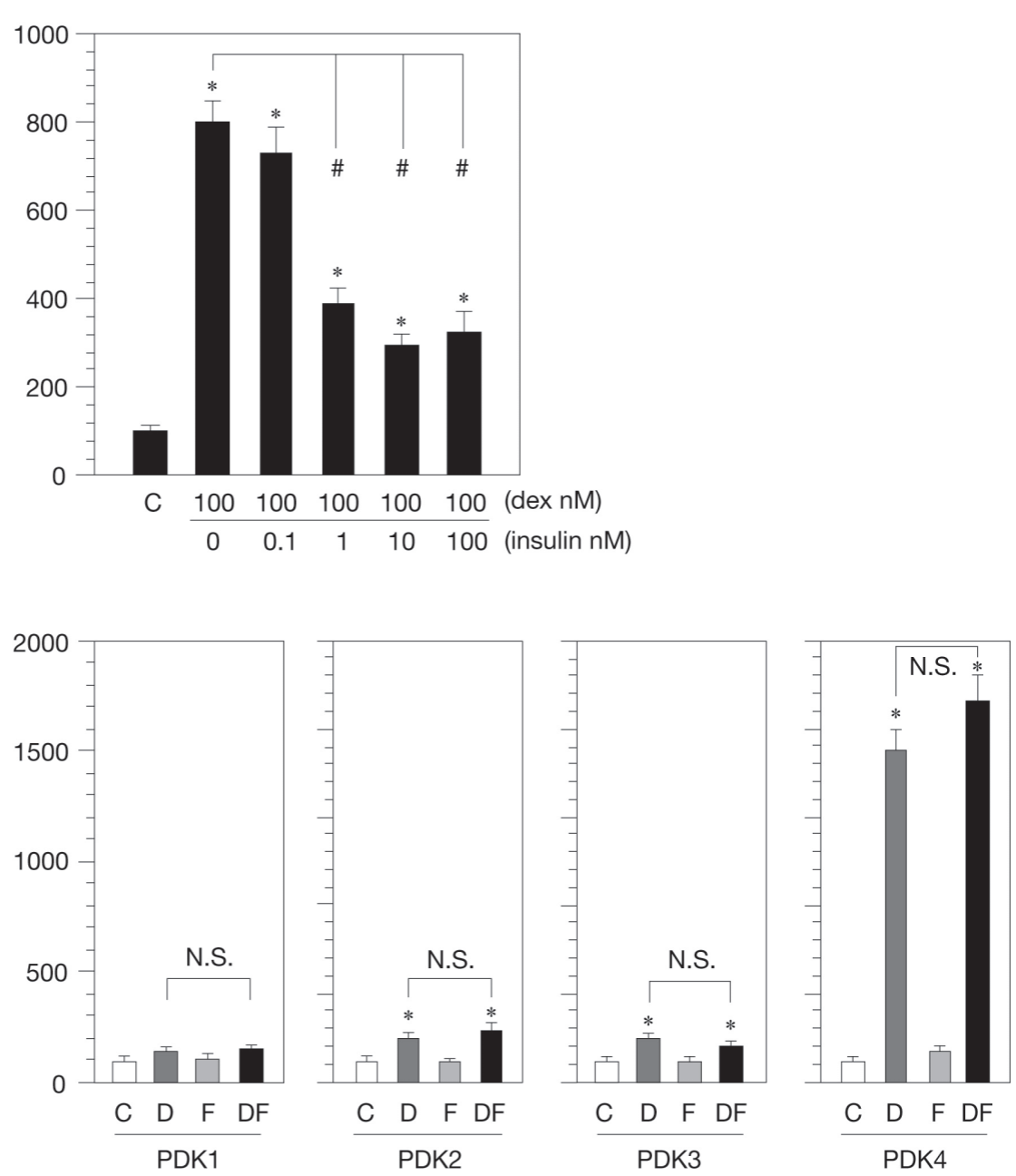

Fig. 5. Combined effects of glucocorticoid and insulin/forskolin on the transcriptional activity of PDKs. A. HepG2 cells were transfected transiently with each test plasmid and treated with dexamethasone (D, $100 \mathrm{nM}$ ) and/or insulin (I, $10 \mathrm{nM}$ ) for $12 \mathrm{~h}$. B. HepG2 cells were transfected as above and treated with dexamethasone (dex, $100 \mathrm{nM})$ and various doses of insulin (0.1$100 \mathrm{nM}$ ) for $12 \mathrm{~h}$. C. HepG2 cells were transfected as above and treated with dexamethasone (D, $100 \mathrm{nM}$ ) and forskolin (F, 10 $\mu \mathrm{M})$ for $12 \mathrm{~h}$. Each value is shown as a percentage of the control value (C). RLU, Relative Light Unit. ${ }^{*} P<0.05$ vs. control. \# $P<0.05$ vs. dexamethasone alone. N.S., not significant. 
A

Promoter Activity

(RLU; \% control)
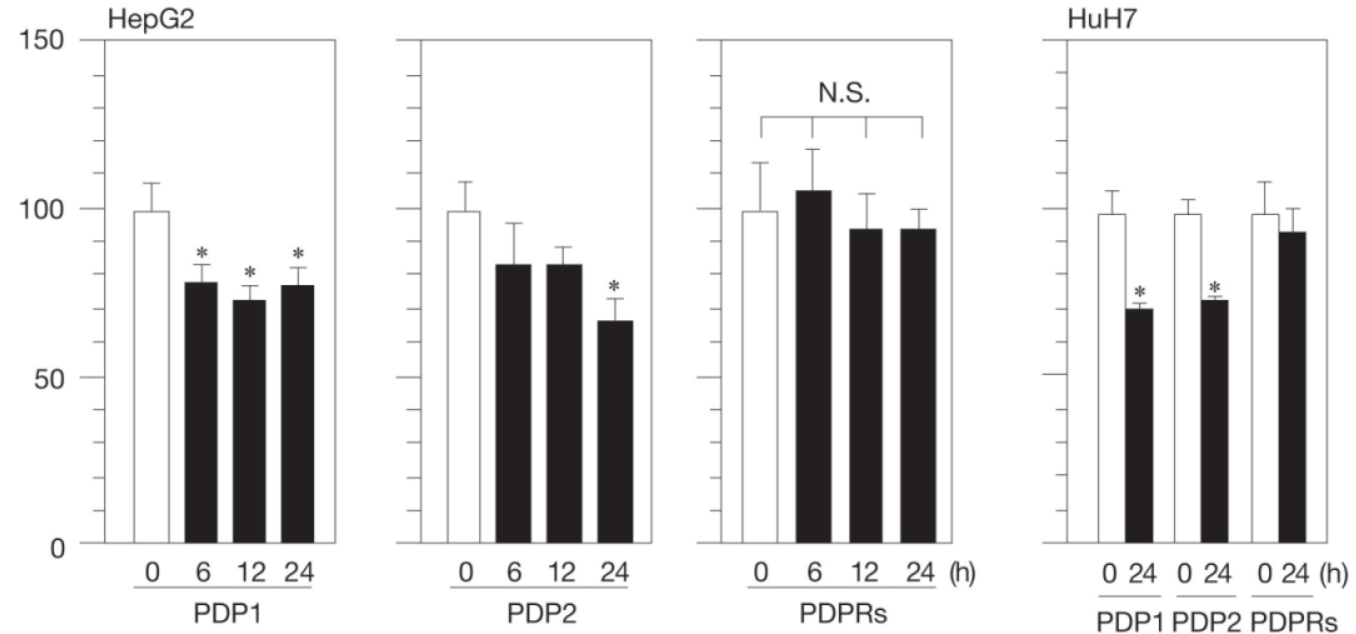

B
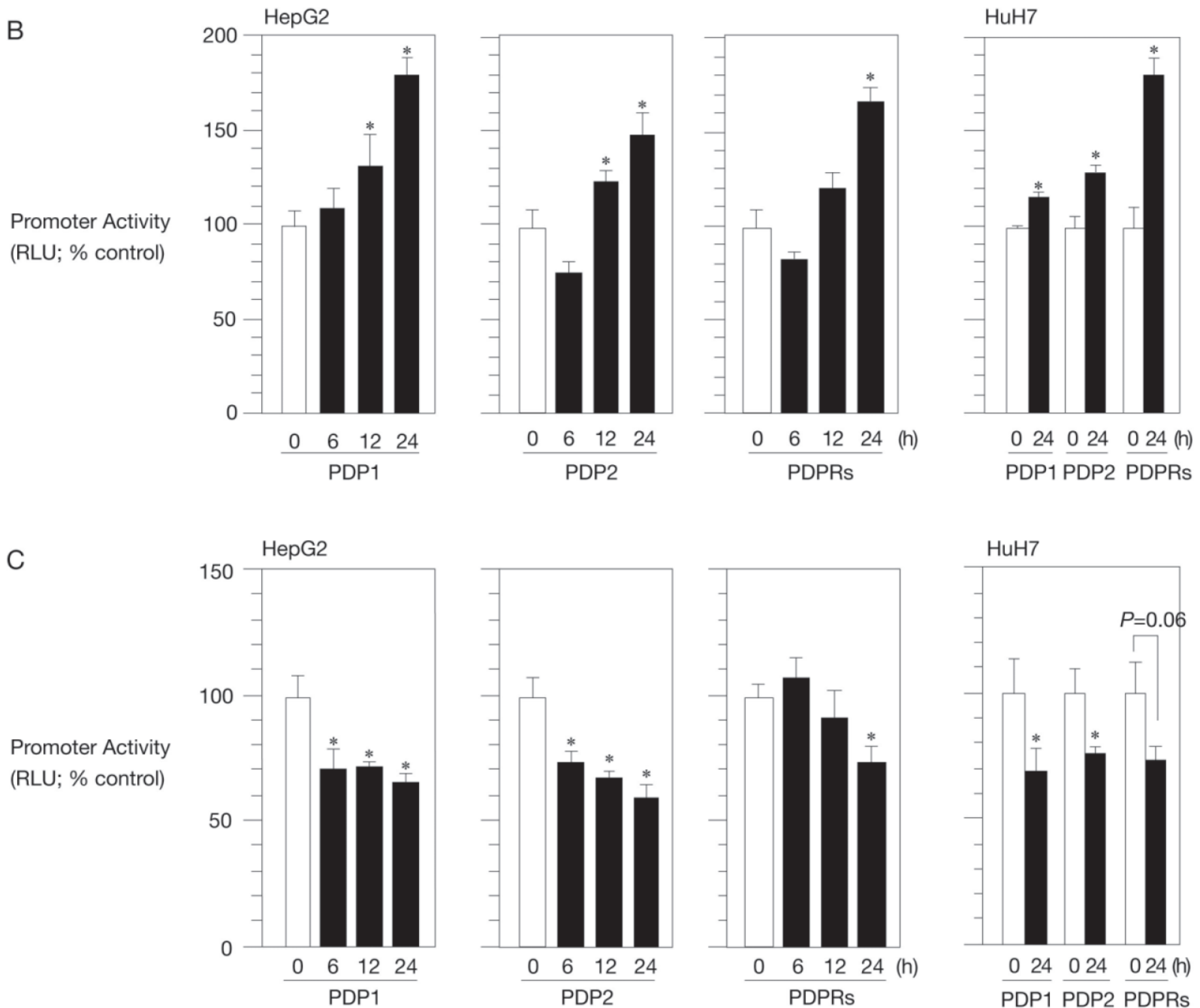

Fig. 6. Time-course effects of glucoregulatory hormones/factors on the transcriptional activity of PDPs. A-C. HepG2 and HuH7 cells were transfected transiently with each test plasmid and treated with dexamethasone (100 nM) (A), insulin (10 nM) (B), or forskolin $(10 \mu \mathrm{M})(\mathrm{C})$, for up to 6-24 h (HepG2) or $24 \mathrm{~h}(\mathrm{HuH7})$. Each value is shown as a percentage of the value at time zero (0). RLU, Relative Light Unit. $* P<0.05$ vs. value at time zero. N.S., not significant. 
Combined effects of the glucoregulatory hormones on the transcriptional activity of each isoform of PDP genes

We finally examined the combined effects of the glucoregulatory hormones/factors on the transcriptional activity of each PDP isoenzyme genes. Insulin (10 $\mathrm{nM}, 12 \mathrm{~h}$ ) alone again showed stimulatory effect on the transcription of PDP1, 2 and PDPRs. However, dexamethasone (100 nM, $12 \mathrm{~h}$ ) dampened or cancelled the positive effect of insulin (Fig. 7A). Similar and more pronounced antagonizing effects were observed when forskolin $(10 \mu \mathrm{M}, 12 \mathrm{~h})$ was simultaneously used with insulin (Fig. 7B).

\section{Discussion}

In this study, we comprehensively surveyed the effects of glucoregulatory hormones such as glucocorticoid, insulin and glucagon/cAMP, on the transcriptional regulation of the key glycolytic enzymes (GK, PFK1, LPK), and the regulatory enzymes (PDKs and PDPs) that are involved in the regulation of PDC. We found that glucocorticoid is involved in the regulation of most of the genes at the transcription level as well as the latter two hormones, and that each hormone examined seems to play a differential role in the regulation of the glycolytic enzyme genes. We also found that, in addition to the positive effect of glucocorticoid on all the PDK isoforms, the hormone has negative effects on PDP1 and 2 isoform genes. Forskolin, used as a surrogate of glucagon, also showed significant inhibitory effects on all the PDP isoforms. Based on our results, we assume that, at the transcriptional level, 1) glucocorticoid exerts a lipogenic effect via a positive effect on PFK1 and LPK; 2) insulin has more pronounced effects on the lipogenic pathway not only by exerting a positive effect on glycolytic enzymes but also by enhancing PDC activity through both negative effects on glucocorticoid-stimulated PDKs and positive effects on PDPs expression; 3) glucagon/cAMP facilitate gluconeogenesis through a different mechanism from that of glucocorticoid, i.e., one which inhibits PDC activity not by stimulating PDKs but by inhibiting PDPs expression. Altogether, our data strongly suggest that all the glycolytic enzymes and PDKs/PDPs are molecular targets of glucoregulatory hormones at the transcriptional level.

Regarding the regulation of glycolytic enzyme gene transcription, insulin showed a positive effect on the transcription of all the key glycolytic genes, as shown in the previous studies [11]. This is in accordance with the well-accepted concept that insulin is a representative anabolic hormone, and plays an indispensable role in the glycolysis/lipogenesis [12, 13]. The time-course effect, however, showed that the effect of insulin on GK and PFK was not as rapid as the physiologically recognized metabolic effect of insulin. This suggests that the short-term metabolic effect of insulin is mediated via well-known allosteric changes of the enzyme proteins, and the observed genomic effect is involved in the long-term adaptative effect in response to the nutritional state of the organism.

When compared with insulin, glucocorticoid clearly showed different effects on glycolytic enzyme gene transcription; we found a suppressive effect on GK, but stimulatory effects on PFK1 and LPK. Among the observed effects, the one on GK is reasonable, because glucocorticoid is a gluconeogenic hormone, and is known to stimulate the expression of glucose-6-phosphatase [14], which has the opposite effect of GK and facilitates the release of glucose from the liver. In addition, the inhibitory effect of glucocorticoid on GK expression might partly be responsible for the hepatic insulin resistance, which is known to occur in glucocorticoid excess. Interestingly, however, glucocorticoid showed stimulatory effects on the transcription of PFK1 and LPK genes, as insulin did, and even had an additive effect with insulin on LPK gene transcription. This suggests that glucocorticoid and insulin are antagonistic at the upstream level of glycolysis but at the downstream level of the pathway the two hormones are rather cooperative. Indeed, glucocorticoid excess is known to facilitate triglyceride synthesis, resulting in the occurrence of abdominal obesity and/or hepatic steatosis $[9,15]$. Although the molecular mechanism responsible for the combined effects is not clear in this study, our data support the classical concept that glucocorticoid is basically an anabolic hormone for fatty acid synthesis [16].

Concerning the regulation of PDKs/PDPs, our data also suggest the presence of complex regulatory mechanisms by glucoregulatory hormones at the transcriptional level. The PDC complex is known to be phosphorylated and inactivated during a fasting state to suppress glycolysis, and the positive effects of glucocorticoid on PDKs gene expression may be responsible for the long-term regulation [17]. The stimulato- 
A

(RLU; \% control)

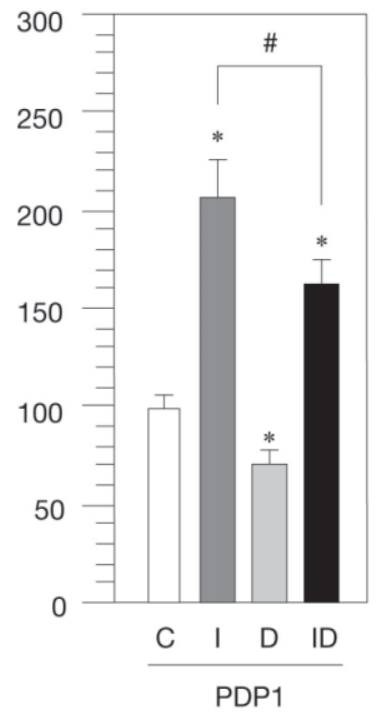

B

Promoter Activity

(RLU; \% control)

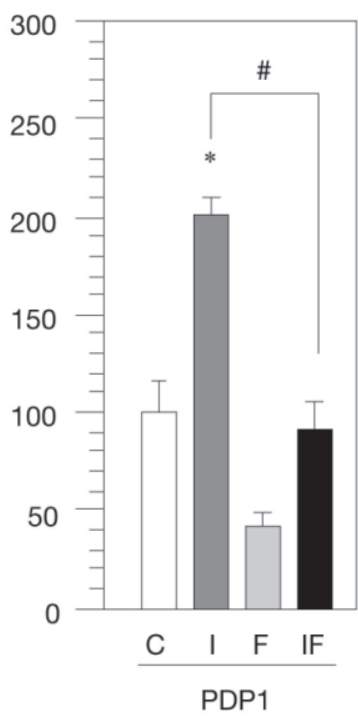

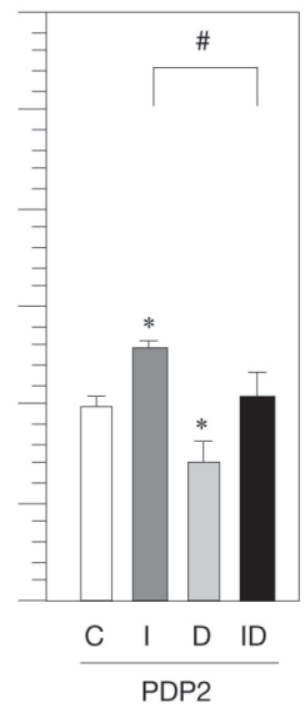
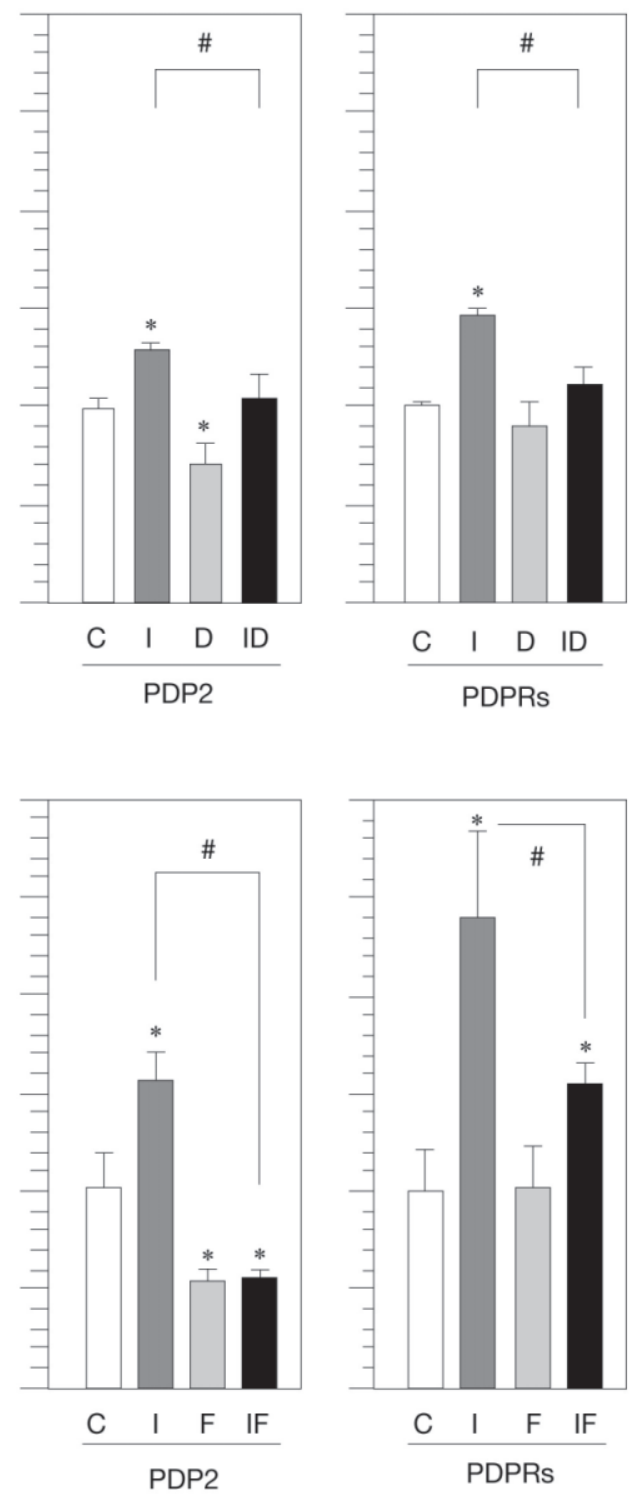

Fig. 7. Combined effects of insulin and dexamethasone/forskolin on the transcriptional activity of PDPs. A. HepG2 cells were transfected transiently with each test plasmid and treated with insulin (I, $10 \mathrm{nM}$ ) and/or dexamethasone (D, $100 \mathrm{nM})$ for $12 \mathrm{~h}$. B. HepG2 cells were also transfected as above and treated with insulin $(10 \mathrm{nM})$ and forskolin $(\mathrm{F}, 10 \mu \mathrm{M})$ for $12 \mathrm{~h}$. Each value is shown as a percentage of the control value (C). RLU, Relative Light Unit. ${ }^{*} P<0.05$ vs. value at time zero. \# $P<0.05$ vs. insulin alone.

ry and inhibitory effects of glucocorticoid and insulin, respectively, on PDK4 gene transcription observed in this study is in accordance with the previous reports $[18,19]$. Kwon et al. clarified the molecular mechanism showing that insulin suppresses the PDK4 induction by glucocorticoid through inactivation of the FOXO factors [18]. In addition, Huang et al. observed insulin inhibition on PDK2 expression as well, but failed to show the effect of dexamethasone alone
[19]. In our study, however, we found clear stimulatory effects of glucocorticoid on other PDKs including PDK2 in both HepG2 and HuH7 cells. The apparent discrepancy of glucocorticoid effect may be due to high basal expression level of PDK2 mRNA in Morris hepatoma $7800 \mathrm{C} 1$ cells or to different experimental paradigm. In any event, both our and previous reports support the concept that insulin and glucocorticoid, when both hormones are present, seem to exert oppo- 
site effects on PDC activity via PDKs.

In sharp contrast, in this study, we revealed that insulin stimulates and glucocorticoid and forskolin/cAMP inhibit the promoter activity of PDP genes. The combination experiment also showed the mutual inhibition of the effects of the two hormones/factors. Since PDPs have opposite effects compared with PDKs, i.e., activate PDC by dephosphorylation [6], our data strongly suggest that the two hormones antagonize each other not only at the level of PDKs, but also at that of PDPs, expression. More interestingly, forskolin also inhibited the transcription of PDPs and eliminated the positive effect of insulin. Glucagon secretion is increased during starvation, which stimulates the intracellular cAMP level in hepatocytes and then facilitates gluconeogenesis as well as inhibits glycolysis. Therefore, the physiological role of glucagon in terms of carbohydrate metabolism is similar to that of glucocorticoid. Although forskolin had minimal influence on the basal transcription of all the PDK isoform genes, it showed potent and significant suppressive effects on PDP1, 2, and PDPRs, and significantly dampened the positive effect of insulin. Thus, in contrast to the effect of glucocorticoid via positive effect on PDKs expression, the effect of glucagon/cAMP seems to be mediated by the inhibitory effects on PDPs expression, although both hormones eventually facilitate gluconeogenesis during starvation. The current study is the first report showing the effects of glucagon/cAMP on PDK/PDP regulation at the transcriptional level.

In this study, the effects of glucoregulatory hormones on the transcriptional activity of each gene surveyed were estimated using a reporter gene technology. Luciferase reporter system is very sensitive, and the values obtained is known to reflect the dynamic changes (both increase and decrease) in the transcrip- tional activity of the target gene(s) examined, because of the intracellular short half-life of the luciferase protein. The findings will also be applicable to the identification of the transcription factor(s) involved in the expression of each gene. However, the transcriptional regulation characterized in tumor cell lines in vitro may not be similar to that occurring in vivo. Furthermore, expression levels of genes/proteins are known to be determined not only by transcription but also by mRNA degradation and/or translation. In this sense, further experiments using a whole animal will be necessary to confirm the physiological significance of the data obtained in this study.

Overall, PDC, mediating the conversion from pyruvate to Acetyl-CoA, is a link between glycolysis and either lipogenesis, gluconeogenesis, or further oxidation in the tricarbonic acid cycle, and thus can be a target of a variety of glucoregulatory hormones including glucocorticoid, insulin, and glucagon/cAMP. The comprehensive examination of the effects of these hormones on all the PDKs and PDPs gene transcription in this study revealed that each hormone has different target gene(s). Nonetheless, the net results seem to be that gluconeogenic hormones like glucocorticoid and glucagon/ cAMP inhibit PDC via phosphorylation, whereas an anabolic hormone like insulin stimulates PDC by dephosphorylation. Therefore, PDC is regarded as a metabolic switch for selecting gluconeogenesis during a fasting state or for glycolysis/lipogenesis during a fed state [20].

\section{Acknowledgements}

This work was supported in part by the Kochi University President's Discretionary Grant and a Research Grant from the Eli Lilly Japan.

\section{References}

1. Sugden MC, Holness MJ (1994) Interactive regulation of the pyruvate dehydrogenate complex and the carnitine palmitoyltransferase system. FASEB J 8: 54-61.

2. Sugden MC, Holness MJ (2003) Recent advances in mechanisms regulating glucose oxidation at the level of the pyruvate dehydrogenase complex by PDKs. Am J Physiol Endocrinol Metab 284: E855-E862.

3. Roche TE, Hiromasa Y (2007) Pyruvate dehydrogenase kinase regulatory mechanisms and inhibition in treating diabetes, heart ischemia, and cancer. Cell Mol
Life Sci 64: 830-849.

4. Patel MS, Korotchkina LG (2001) Regulation of mammalian pyruvate dehydrogenase complex by phosphorylation: complexity of multiple phosphorylation sites and kinases. Exp Mol Med 33: 191-197.

5. Holness MJ, Sugden MC (2003) Regulation of pyruvate dehydrogenase complex activity by reversible phosphorylation. Biochem Soc Trans 31: 1143-1151.

6. Roche TE, Baker JC, Yan X, Hiromasa Y, Gong X, Peng T, Dong J, Turkan A, Kasten SA (2001) Distinct 
regulatory properties of pyruvate dehydrogenase kinase and phosphatase isoforms. Prog Nucleic Acid Res Mol Biol 70: 33-75.

7. Patel MS, Korotchkina LG (2006) Regulation of the pyruvate dehydrogenase complex. Biochem Soc Trans 34: 217-222.

8. Barthel A, Schmoll D (2003) Novel concepts in insulin regulation of hepatic gluconeogenesis. Am J Physiol Endocrinol Metab 285: E685-E692

9. Newell-Price J, Bertagna X, Grossman AB, Nieman LK (2006) Cushing's syndrome. Lancet 367: 16051617.

10. Aoki Y, Iwasaki Y, Katahira M, Oiso Y, Saito H (1997) Regulation of the rat proopiomelanocortin gene expression in AtT-20 cells. I: Effects of the common secretagogues. Endocrinology 138: 1923-1929.

11. Wu C, Khan SA, Lange AJ (2005) Regulation of glycolysis-role of insulin. Exp Gerontol 40: 894-899.

12. Foufelle F, Ferré P (2002) New perspectives in the regulation of hepatic glycolytic and lipogenic genes by insulin and glucose: a role for the transcription factor sterol regulatory element binding protein-1c. Biochem $J$ 366: 377-391.

13. Raghow R, Yellaturu C, Deng X, Park EA, Elam MB (2008) SREBPs: the crossroads of physiological and pathological lipid homeostasis. Trends Endocrinol Metab 19: 65-73.

14. Vander Kooi BT, Onuma H, Oeser JK, Svitek CA, Allen SR, Vander Kooi CW, Chazin WJ, O’Brien RM
(2005) The glucose-6-phosphatase catalytic subunit gene promoter contains both positive and negative glucocorticoid response elements. Mol Endocrinol 19: 3001-3022.

15. Rockall AG, Sohaib SA, Evans D, Kaltsas G, Isidori AM, Monson JP, Besser GM, Grossman AB, Reznek RH (2003) Hepatic steatosis in Cushing's syndrome: a radiological assessment using computed tomography. Eur J Endocrinol 149: 543-548.

16. Brindley DN (1981) Regulation of hepatic triacylglycerol synthesis and lipoprotein metabolism by glucocorticoids. Clin Sci (Lond) 61: 129-133.

17. Wu P, Blair PV, Sato J, Jaskiewicz J, Popov KM, Harris RA (2000) Starvation increases the amount of pyruvate dehydrogenase kinase in several mammalian tissues. Arch Biochem Biophys 381: 1-7.

18. Kwon H-S, Huang B, Unterman TG, Harris RA (2004) Protein kinase B- $\alpha$ inhibits human pyruvate dehydorgenase kinase-4 gene induction by dexamethasone through inactivation of FOXO transcription factors. Diabetes 53: 899-910.

19. Huang B, Wu P, Bowker-Kinley MM, Harris RA (2002) Regulation of pyruvate dehydrogenase kinase expression by peroxisome proliferator-activated receptor-alpha ligands, glucocorticoids, and insulin. Diabetes 51: 276-283.

20. Randle PJ (1986) Fuel seleciton in animals. Boochem Soc Transact 14: 799-806. 